\title{
Double HEVC Compression Detection with the Same QPs Based on the PU Numbers
}

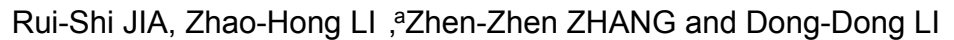 \\ school of Electronic and Information Engineering, Beijing Jiao tong University, Beijing, 100044 \\ \{15120012, zhhli2, 11111053, 14120011\}@bjtu.edu.cn \\ a Corresponding author: Zhaohong Li, zhhli2@bjtu.edu.cn
}

\begin{abstract}
Double HEVC compression detection is of great importance in video forensics. However, effective detection algorithm based on the same Qps is rarely reported. In this paper, a novel method based on the same Qps is applied in dealing with double HEVC compression detection. Firstly, the numbers of PU blocks with the size of $4 \times 4$ in each I frame is extracted during the codec procedures when the video is compressed with the same Qps. Then, calculate the standard deviation of $4 \times 4$ PU blocks difference (SDoPU) before and after the compression. Finally, select the appropriate threshold for compression testing classification according to the SDoPU statistical feature. By performing numerical experiments, We prove that the proposed algorithm is of high classification accuracy for detecting double HEVC compression.
\end{abstract}

\section{Introduction}

Nowadays, with the advent of the era of network and multimedia information, digital video has become an indispensable part of our life. However, as the high-speed development of the video editing software such as Adobe Premiere, the authenticity and integrity of videos suffered severe damage. Therefore, video tampering forensics becomes a necessary link to ensure the authenticity of copyright problem and surveillance videos. Double compression detection as an important detection means of video forensics technology and therefore has become a hot spot in the field of information security.

So far, existing methods are effective for double compression detection. $\mathrm{Su}$ and $\mathrm{Xu}$ detect double compression by studying the distribution of quantized DCT coefficients for MPEG video in [1,2]. By judging whether the probability distribution of the first digits of the non-zero MPEG quantized AC coefficients will be disturbed, Chen in [3] distinguish the occurrence of the double compression. Jiang in [4] use Markov statistics to classify double compression. Huang detect double MPEG compression according to the change of the numbers of DCT coefficients for the primary and secondary compression. While, above algorithms are all for the compressed video before the coding standard HEVC. References $[5,6]$ proposed the methods based on the cooccurrence of DCT coefficients, [7] use the odd-even feature of the DCT coefficients, and references $[5,6,7]$ are based on the latest coding standard.

There is a lack of effective algorithm dealing with double compression detection based on the same Qps,
Double HEVC compression detection with the same Qps is less. Nevertheless, as the latest coding standard, HEVC video coding standard will play an increasingly important role in $\mathrm{Hd}$ and High-definition video in the future. Therefore, double HEVC compression detection with the same Qps is of particular practical significance. In this paper, a novel method based on the same Qps for double HEVC compression detection is proposed. The main structure of this paper is divided into four parts, Section 2 introduces PU division pattern of HEVC video coding standard, Section 3 gives a description of the specific algorithm, while the part of video database and the experimental results is given in Section 4, we conclude this paper in Section 5 and list some promising directions for future work.

\section{Materials and Methods}

\subsection{The Introduction of PU Types}

As the latest generation of video coding standard, HEVC adopts much more flexible coding block structure, introduces residual coding and the quadtree partitioning structure. CU for coding unit, its size set to $8 \times 8,16 \times 16$, $32 \times 32$ and $64 \times 64$, of course, $\mathrm{CU}$ can be divided into smaller CU unit. Figure 1 shows the $\mathrm{CU}$ partitions and its quadtree structure. As shown in Figure1 (a), HEVC traverses the $\mathrm{CU}$ in the way of $\mathrm{Z}$ scanning when coding it and the number in the diagram block shows the coding sequence. Figure 1(b) shows the corresponding quad tree structure of $\mathrm{CU}$, among them the "solid black spots"on 
behalf of the nodes can be broken down while the hollow ones can't, and there is no need to continue to break down when the size of $\mathrm{CU}$ is $8 \times 8$.

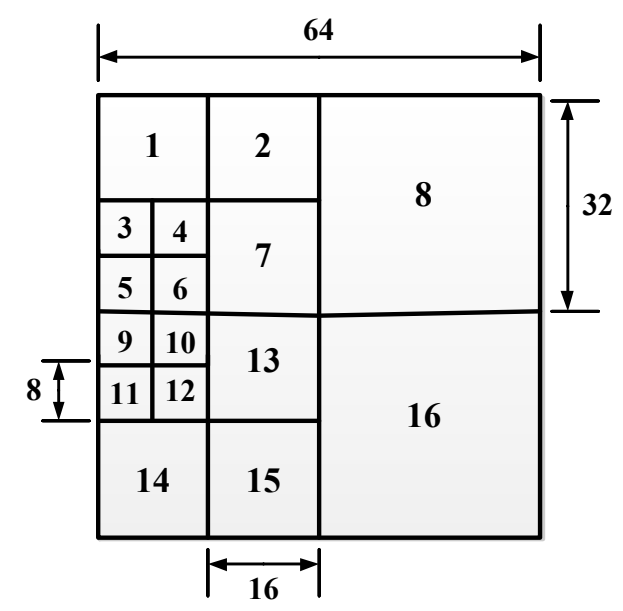

(a) CU Partitions.

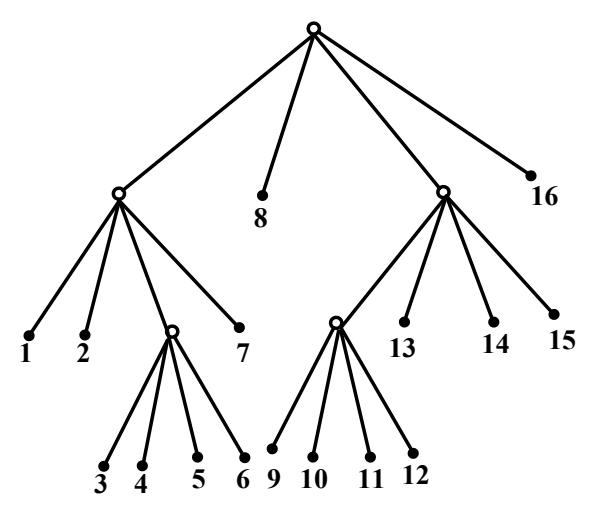

(b) Quadtree Structure

Figure 1. CU Partitions and Its Quadtree Structure.

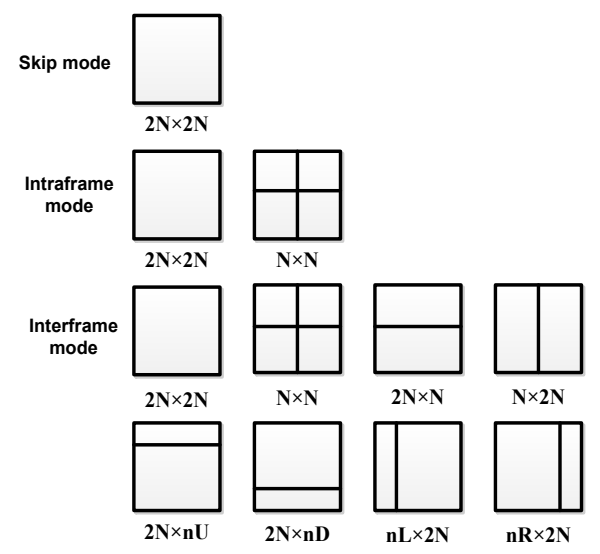

Figure 2. PU Types Under Different Prediction Mode.

PU is the basic unit of the frame and interframe prediction, each internal structure of $\mathrm{CU}$ can be divided into one or more PU, so can look $\mathrm{CU}$ as the outermost layer of the framework and the size of the PU can't larger than $\mathrm{CU}$, its size can be $4 \times 4,8 \times 8,16 \times 16,32 \times 32$ and $64 \times 64$. Assuming that the size of the coding unit $\mathrm{CU}$ is $2 \mathrm{~N} \times 2 \mathrm{~N}$, the $\mathrm{CU}$ can be divided into multiple prediction units PU depending on the prediction mode. HEVC provides eight kinds of $\mathrm{PU}$ division structure as shown in Figure 2. The size of the PU only can be $2 \mathrm{~N} \times 2 \mathrm{~N}$ when the forecast model is Skip, about the frame prediction, the PU size can be $2 \mathrm{~N} \times 2 \mathrm{~N}$ and $\mathrm{N} \times \mathrm{N}$, While 8 types of classification are all available at the interframe prediction, $2 \mathrm{~N} \times \mathrm{nU}, 2 \mathrm{~N} \times \mathrm{nD}$, and $\mathrm{nR} \times 2 \mathrm{~N}$ is asymmetric mode, as optional mode, can control its open or close in encoding configuration file.

\subsection{Feature Extraction}

Our algorithm is based on the statistics features of the numbers of $4 \times 4$ PU numbers. Therefore, first of all we should extract the PU types of each video.

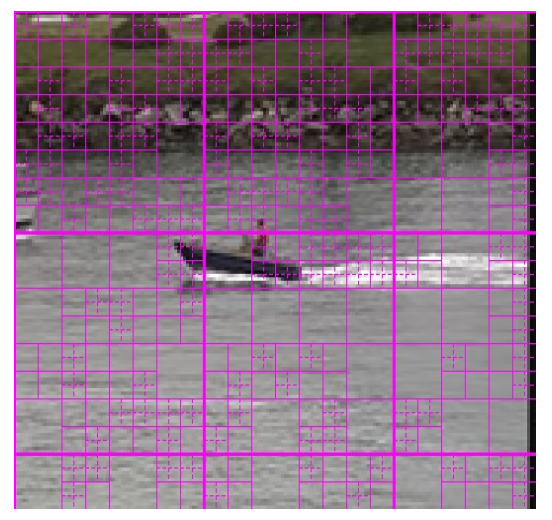

(a) Pu Types In Qp22

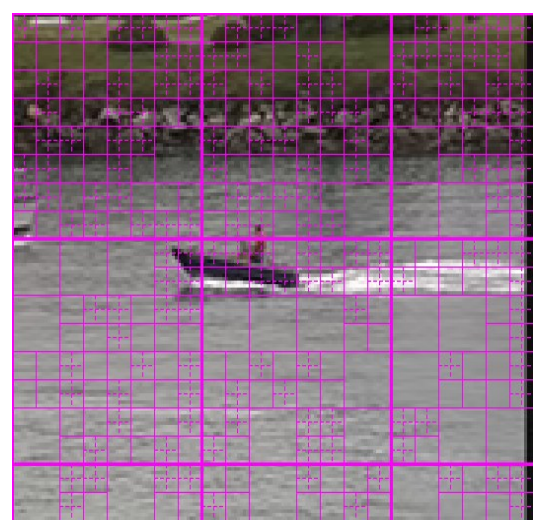

(b)Pu Types In Qp22_22

Figure 3. Pu Types for the First i Frame of the Compressed

Video Part1_Coastguard in QP22 and QP22_22.

Figure 3 shows the PU types for the first I frame of the compressed video part1_coastguard in Qp22 and Qp22_22, Figure 3(a) shows the PU types for the first I frame of part1_coastguard in Qp22 and Figure 3(b) in Qp22_22, Among them, the boundary of the thick solid lines show the maximum size $(64 \times 64)$ of $\mathrm{CU}$, And so on, the dotted line said the smallest size $(4 \times 4)$ for PU blocks.

Figure 4 shows the numbers of $4 \times 4,8 \times 8$ and $16 \times 16$ PU blocks for this two PU types. the curves with the triangle represent the numbers of $16 \times 16$ PU blocks for the under test video and it's one compressed ones, and 
the curves with the circle on behalf of the $8 \times 8$ PU blocks, we can clearly see that they are almost consistent. While the two curves with stars have certain gap, it shows that the numbers of the $4 \times 4$ PU blocks is different. So we can choose the change of $4 \times 4$ PU blocks numbers as the method to detect the double compression videos. Figure 5 is the standard deviation of $4 \times 4$ PU blocks difference (SDoPU) before and after the compression for single and Double compressed videos.

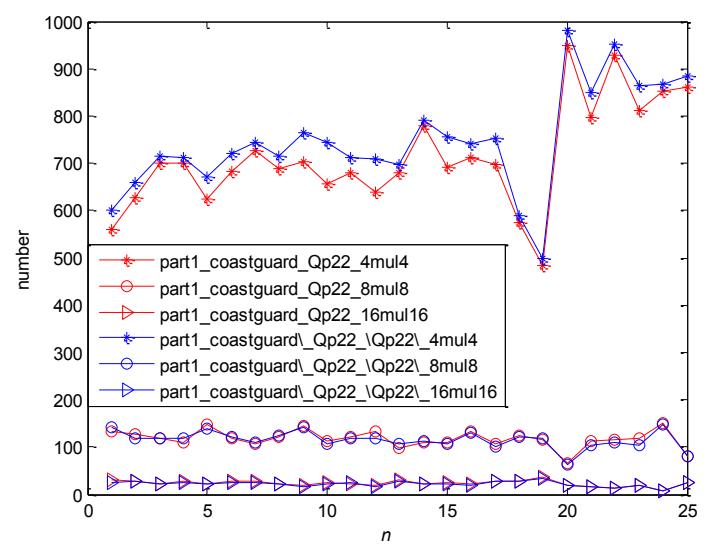

Figure 4. The Numbers of $4 \times 4,8 \times 8$ and $16 \times 16 \mathrm{Pu}$ Blocks for

The Video Part1_Coastguard Based on QP22 and QP22_22

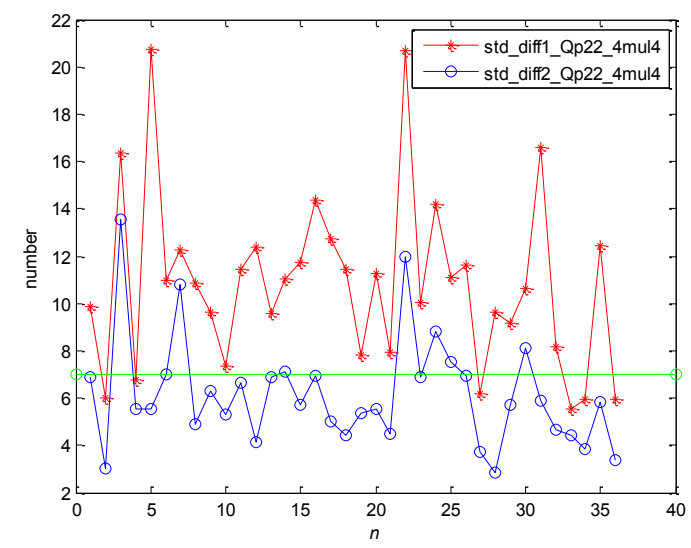

Figure 5. The Standard Deviation of $4 \times 4$ PU Blocks Difference (SDoPU) before and after the Compression for Single and Double Compressed Videos.

In Figure 5, we can see there are three different curves. The curve with the stars represents the values of SDoPU for single compressed videos based on Qp22, and the curve with the circle on behalf of the double compressed videos based on Qp22_22, Qp22_22 means a single compressed video is compressed twice with the same Qp22. Apparently, the two curves have big difference in values. We decide a appropriate threshold to classify them according to their statistics features as the green line shows.

\subsection{Proposed Detection Scheme}

For a given video under test, obviously we can't know whether it's a single or double compression ones. But we can draw the curves of the numbers for the $4 \times 4$ PU blocks, and distinguish the double compression according to the statistics features. The main process of the algorithm is as follows: First of all, decompress the under test video and extract the quantization parameter Qp. Secondly, compress the video with the same Qps extracted from the under test video. Thirdly, check the PU types of each I frame for the primary and secondary compressed videos using the code stream analysis software GtiHEVCAnalyzer [8] of the open source HEVC and draw the curve of the numbers for the $4 \times 4 \mathrm{PU}$ blocks for them. Then, calculate the standard deviation of $4 \times 4$ PU blocks difference (SDoPU) before and after the compression. Finally, select the appropriate threshold for compression testing classification according to the SDoPU statistical feature, namely, according to the two different curves, we select a general range of the threshold value, then select a straight line that the most of points on the two curves distribute on both sides of this line. Figure 6 is the flow chart of the algorithm.

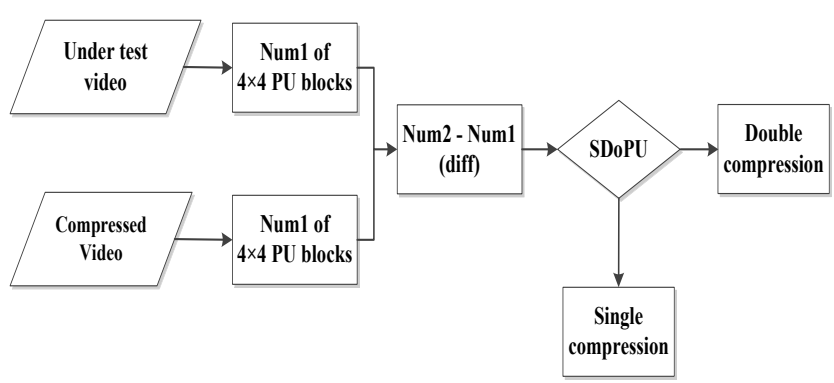

Figure 6. The Flow Chart of the Algorithm.

\section{Results and Discussions}

\subsection{Video Database}

In our experiments, with the resolution of $176 \times 144$ pixels, 36 original YUV sequences of QCIF format available at [9] were used, It's frame rate is $30 \mathrm{f} / \mathrm{s}$ and these videos have various kinds of content, such as people, scene, news, etc. Using the basic HM10.0 class [10], the experiment realizes the HEVC standard codec process, and the GOP structure is IPPP. To ensure the quality of these videos and the experiment better,we set the Qp as $22,24,26,28$ and 32.

\subsection{The Test Standard and Experiment Result}

In this paper, $A R$ (accuracy) is adopted as the evolution standard for detection rate, as shown in formula (1):

$$
A R=(P R+T N R) 2
$$

Where $T P R=T P \quad / \quad(T P+F N)$ and $T N R=T N$ / $(T N+F P), T N$ ( true negative ) represents a single 
compressed video was found as single compressed, TP(true positive) for double compressed video was convicted as single compressed, $F N$ (false negative) for double compressed video was classified as single compressed video, and $F P$ (false positive) for a single compressed video was detected as double compressed video. Table 1 shows the detection rate of double HEVC compression.

Table 1. Detection Rate of double HEVC Compression .

\begin{tabular}{|c|c|c|c|c|c|}
\hline $\begin{array}{c}\text { Detection } \\
\text { rate }\end{array}$ & Qp22 & Qp24 & Qp26 & Qp28 & Qp32 \\
\hline $\begin{array}{c}\text { TNR } \\
\text { (SDoPU) }\end{array}$ & 0.8472 & 0.7778 & 0.8333 & 0.8333 & $\begin{array}{c}0.805 \\
6\end{array}$ \\
\hline $\begin{array}{c}\text { TRP } \\
\text { (SDoPU) }\end{array}$ & 0.8611 & 0.8056 & 0.7222 & 0.8611 & $\begin{array}{c}0.888 \\
9\end{array}$ \\
\hline AR & 0.8472 & 0.7917 & 0.7778 & 0.8472 & $\begin{array}{c}0.847 \\
3\end{array}$ \\
\hline
\end{tabular}

As shown in Table1, we set five quantization parameters Qps to compressed videos. When the quantization parameter is Qp32, the AR of the proposed method is 0.8473 for classifying firstly-compressed and doubly-compressed videos; this parameter achieves the best detection results. As the latest coding standard, there are few effective methods for HEVC video detection, The result demonstrates our algorithm is promising.

\section{Conclusions}

With the development of the advanced video editor, the authenticity and integrity of the videos can't be guaranteed anymore. In this paper, we conduct a research to detect double HEVC compression and proposed a novel method based on the same Qps. We extract the PU types of the under test videos and it's primary and secondary compressed ones, By calculating the SDoPU of $4 \times 4$ PU blocks, a set of statistics features have been extracted to classify double HEVC compression videos. Since there are few effective algorithms focusing on the detection for double HEVC compression, the performance of our proposed algorithm is promising, and we will further research to improve our algorithm in the future.

\section{Acknowledgment}

We would like to thank Xian Yang and Yawei Yuan for their help and kindly suggestions. This work was supported in part by the Fundamental Research Funds for the Central Universities (W15JB00280), in part by SRF for ROCS, SEM (W15C300020), in part by A Project Funded by the Priority Academic Program Development of Jiangsu Higer Education Institutions (PAPD), and in part by Jiangsu Collaborative Innovation Center on Atmospheric Environment and Equipment Technology (CICAEET).

\section{References}

[1] Tanfeng Sun, wan wang, and Xinghao Jiang, "Exposing video forgeries by detecting MPEG double compression," in Acoustics, Speech and signal Processing (ICASSP), 2012 IEEE international Conference on IEEE, 2012, pp. 1389-1392.

[2] JUNYU XU, YUTING SU,QINGAHONG LIU. Detection of double MPEG-2 compression based on distribution of DCT coefficients [J]. International Journal of Pattern Recognition \& Artificial Intelligence, 2013, 27(1):155-167.

[3] Wen Chen and Yun Q shi, "Detection of double MPEG compression based on first digit statistics," in Digital Watermarking, pp. 16-30. Springer,2009.

[4] Jiang, X, Wang, W, Sun, T, et al. Detection of Double compression in MPEG-4 Videos Based on Markov Statistics, Singal Procession Letters, 2003, 20, (5), pp. 447-450.

[5] Huang M, Wang R, Xu J, et al. Detection of Double Compression for HEVC Videos Based on the Cooccurrence Matrix of DCT Coefficients[M]// DigitalForensics and Watermarking. Springer International Publishing, 2015.

[6] Jieyuan Chen, Xinghao Jiang, tangfeng Sun, Peisong He, Shilin Wang. DETECTING DOUBLE MPEG COMPRESSION WITH THE SAME QUANTISER SCALE BASED ON MBM FEATURE. Conference paper. ICASSP.2016.7472040.

[7] Huang M L, Wang R D, Xu J, et al. Detection of double compression in HEVC videos based on the statistical characteristic of DCT coefficients[J]. Guangdianzi Jiguang/journal of optoelectronics laser, 2015, 26(4):733-739..

[8] SourceForge.net,"GtilHEVCAnalyzer", https://sourceforge.net/projects/gtilhevcanalyzer/.

[9] 'YUV video sequences', http://trace.eas.asu.edu/yuv/index.html, accessed 2 August 2015.

[10] JVT-VC Reference Software version

HM-10.0 Available

on http://download.csdn.net/detail/amymayadi/7903385 